Journal of Case Reports 2018;8(3):187-189

\title{
Community Acquired VRSA Pneumonia: Need for Alarm
}

\author{
Shalini Akunuri, Shraddha Pandey \\ Department of Pediatrics, Lotus Hospital for Women and Children, Visakhapatnam, Andhra Pradesh, India.
}

\author{
Corresponding Author: \\ Dr. Shalini Akunuri \\ Email: akunurishalini@gmail.com
}

This is an Open Access article distributed under the terms of the Creative Commons Attribution License (creativecommons.org/ licenses/by/3.0).

Received Accepted

Published

December 9, 2017

July 7, 2018

August 5, 2018

\begin{abstract}
Background: Vancomycin resistant Staphylococcus aureus (VRSA) is an important multi-drug resistant organism of public health importance. Most strains have been isolated in hospitalized patients. However, there are rare case reports from community as well. Case Report: We report a case of community acquired pneumonia with empyema due to vancomycin and teicoplanin resistant Staphylococcus aureus. Conclusion: The present case is a community acquired infection and re-emphasizes the need for rationale antibiotic policy and appropriate infection control measures.
\end{abstract}

Keywords: Community-Acquired Infections, Empyema, Staphylococcus aureus, Teicoplanin, Vancomycin Resistance.

\section{Introduction}

Emergence of Staphylococcus aureus (S. aureus) with diminished susceptibility was anticipated when vancomycin resistant enterococci (VRE) was initially described. Infection with vancomycin resistant $S$. aureus (VRSA) is rare and is usually encountered in healthcare associated settings. Common predisposing factors for VRSA include ongoing or recent dialysis, bacteremia related to central venous catheter or prosthetic graft material and prolonged vancomycin exposure (6-18 weeks) in the preceding 3-6 months. We report a case of community acquired pneumonia (CAP) with empyema due to VRSA.

\section{Case Report}

Two year girl presented with high grade fever for 13 days and breathlessness for six days. She was treated as an outpatient by her primary pediatrician with oral antibiotics for five days, failing which intravenous antibiotics were initiated. At presentation, she was febrile, breathless, with absent air entry over left chest and stony-dull percussion note. Chest-radiograph showed homogenous opacity of left lung fields with tracheal deviation to the opposite side. Ultrasonography chest revealed massive pleural effusion with internal echoes. Her septic work-up was positive. Pleural fluid analysis was suggestive of exudate. She was started on empiric antibiotics with ceftriaxone and vancomycin along with tube thoracocentesis and intrapleural fibrinolytics.

By day-three of hospitalization, pleural fluid grew $S$. aureus. With Kirby-bauer discdiffusion method, the isolate grew 12-mm zone of inhibition around the vancomycin disc suggesting that the isolate had decreased susceptibility to vancomycin. MIC of vancomycin was $32 \mu \mathrm{g} / \mathrm{mL}$ as determined by broth-microdilution. The isolate was resistant to oxacillin, cefazolin, fluoroquinolones (ciprofloxacin, levofloxacin), aminoglycosides (gentamycin, amikacin), glycopeptides (vancomycin, teicoplanin), erythromycin. Clindamycin was intermediate sensitive; however D-zone disc-diffusion test (D-test) was positive confirming inducible clindamycin resistance. The organism was sensitive to linezolid, tigecycline, rifampin, daptomycin and quinupristin-dalfopristin. 
Initial days of hospitalization were complicated by persistence of high spiking fever and respiratory distress. Antibiotics were changed to linezolid, rifampicin and tigecycline, and decortication was performed. Patient's rapid clinical improvement soon after

\section{Discussion}

S. aureus is an important cause of serious infection in community and hospital settings. In the past 4 decades, there is increasing incidence of methicillin resistant $S$. aureus (MRSA) resulting in frequent use of vancomycin, which is first line drug for MRSA, consequently leading to development of vancomycin resistance due to selective pressure.

The first strain of vancomycin intermediate sensitive $S$. aureus (VISA) was reported from Japan in 1997 [1]. In June 2002, VRSA was detected in a patient from Michigan [2]. Since then there has been increased incidence of resistance to $S$. aureus from different parts of the world. In India, first isolate of VRSA was reported from Kolkata in June 2005 [3]. According to Clinical and Laboratory Standards Institute (CLSI), staphylococci with $\mathrm{MIC} \leq 2 \mu \mathrm{g} / \mathrm{mL}$ are sensitive (VSSA), MIC 4-8 $\mu \mathrm{g}$ / $\mathrm{mL}$ are intermediate sensitive (VISA), and $\mathrm{MIC} \geq 16$ $\mu \mathrm{g} / \mathrm{mL}$ are resistant (VRSA) [4]. The changing susceptibility of staphylococci has led CLSI to lower vancomycin susceptibility breakpoint for S. aureus from $4 \mu \mathrm{g} / \mathrm{mL}$ to $2 \mu \mathrm{g} / \mathrm{mL}$ in 2006 . There is linear relationship between MIC and clinical failure rates [5]. The term glycopeptide resistant S. aureus (GRSA) is more appropriate for isolates resistant to both vancomycin and teicoplanin.

Environmental factors contributing to vancomycin resistance are irrational antibiotic use, over-the-counter availability of drugs without prescription, injudicious use, agriculture, fisheries and animal husbandry, which could result in increased selective pressure of vancomycin [6]. Among the clinical factors, exposure to glycopeptides or vancomycin is the biggest risk factor for VRSA. Peritoneal dialysis and renal failure may also be risk factors [7]. Community acquired MRSA should be suspected when pneumonia develops in a person known to be colonized with MRSA or in those with risk factors such as contact sport participants, injection drug users, those living in crowded conditions, recent influenza-like illness, fulminant course, pulmonary necrosis or empyema, sputum Gram stain showing gram-positive cocci in clusters. Because the patient had fulminant infection and was not hospitalized prior to illness, we presume that she acquired this resistant organism from the community.

The optimal antimicrobial regimen for VRSA is unknown. An appropriate approach is treatment with at least one antimicrobial to which the organism is known to be susceptible by in vitro testing. Teicoplanin as an alternative to vancomycin was short-lived as the bacteria quickly developed resistance due to similarities of glycopeptides. Daptomycin should not be used to treat pneumonia as the drug is inhibited by pulmonary surfactant. Ceftaroline has grampositive (MRSA, VISA and macrolide-resistant $S$. pyogenes) and gram-negative (Enterobacteriaceae) bactericidal activity. Quinupristin-dalfopristin has activity against MRSA and VRSA isolates [8] and is an alternative to vancomycin in children if the isolate is susceptible [9]. Tigecycline has activity against gram positive organisms including MRSA and VRE. It is used for skin and skin structure infection, intra-abdominal infections and CAP. Linezolid is FDA approved for treatment of VRE and MRSA necrotizing infections like skin lesions, fasciitis and pneumonia. Linezolid and clindamycin inhibit exotoxin production in Panton Valentine Leucocidin positive community acquired MRSAs and should be preferred [10]. Rifampicin has bactericidal activity against $S$. aureus and achieves high intracellular levels, in addition to penetrating biofilms. However, because of rapid development of resistance, it should never be used as monotherapy. Telavancin is bactericidal against MRSA, VISA, and VRSA. 
The choice of antibiotics should be based on susceptibility reports, site of infection (endocarditis, urinary tract infection, complicated skin and skin structure infection, catheter related blood stream infections), severity of infection and clinical response to regimen chosen. Empiric use of vancomycin when not indicated should be strongly discouraged, such as routine surgical prophylaxis other than in a patient with lifethreatening allergy to beta-lactam antibiotics, empiric therapy for febrile neutropenia, treating single coagulase-negative, Staphylococcus positive cultures, continued empiric use of presumed infections in patients whose cultures are negative, prophylaxis for colonization of intravascular catheters, selective decontamination of digestive tract, eradication of MRSA colonization, primary treatment of antibiotic-associated colitis, routine prophylaxis for very low birth-weight infants and topical application or irrigation.

\section{Conclusion}

The microbiology laboratory plays a pivotal role in early detection and reporting of VRSA. In addition, close liaison between clinician and microbiologist will help formulate appropriate antibiotic choice and significant reduction in mortality and morbidity. Also the emergence and spread of VRSA can be significantly curtailed if appropriate infection control policies are implemented. The present case is a community acquired infection and re-emphasizes the need for rationale antibiotic policy. This case highlights the need for further studies, microbiological confirmation and need for reporting community acquired VRSA.

Contributors: SA and PS conceptualized the report, did the literature search and took part in the clinical care of the patient. SA drafted the original manuscript. PS reviewed and edited the manuscript. Both authors have read and approved the final version of the manuscript. SA will be guarantor of the case report.

Funding: None; Competing interests: None stated.

\section{References}

1. Hiramatsu K, Hanaki H, Ino T, Yabuta K, Oguri T, Tenover FC. Methicillin-resistant Staphylococcus aureus clinical strain with reduced vancomycin susceptibility. J Antimicrob Chemother. 1997;40:135-136.

2. Chang S, Sievert DM, Hageman JC, Boulton ML, Tenover FC, Downes FP, et al. Infection with vancomycin-resistant Staphylococcus aureus containing Van A resistance gene. N Engl J Med. 2003;348:13421347.

3. Saha B, Singh AK, Ghosh A, Bal M. Identification and characterization of a vancomycin resistant Staphylococcus aureus isolated from Kolkata (South Asia). Journal of Medical Microbiology. 2008;57:72-79.

4. CLSI. Performance standards for antimicrobial susceptibility testing: 22nd information supplement. CLSI document M100-S22. Wayne, PA: Clinical and Laboratory Standards Institute; 2012.

5. Moise Broder PA, Sakoulas G, Eliopoulos GM, Schentag JJ, Forrest A, Moellering RC Jr. Accessory gene regulator group II polymorphism in methicillinresistant Staphylococcus aureus is predictive of failure of vancomycin therapy. Clin Infect Dis. 2004;38:17001705.

6. Tiwari HK, Sen MR. Emergence of vancomycin resistant $\mathrm{S}$ aureus from a tertiary care hospital from northern India. BMC Infect Dis. 2006;6:156-161.

7. Srinivasan A, Dick JD, Peri TM. Vancomycin resistance in Staphylococci. Clin Microbial Rev. 2002;15:430-438.

8. Werner G, Cuny C, Schmitz FJ, Witte W. Methicillinresistant, quinopristin-dalfopristine-resistant Staphylococcus aureus with reduced sensitivity to glycopeptides. J Clin Microbiol. 2001;39:3586.

9. American Academy of Pediatrics. Staphylococcal infections. In: Red Book: 2012 Report of the committee on infectious Diseases, 29 ${ }^{\text {th }}$, Pickering LK. (Ed), American Academy of Pediatrics, Elk Grove Village, IL 2012. pp. 653

10. Micek ST, Dunne M, Kollef MH. Pleuropulmonary complications of Panton-Valentine leukocidinpositive community-acquired methicillin-resistant Staphylococcus aureus: importance of treatment with antimicrobials inhibiting exotoxin production. Chest. 2005;128:2732-2738. 